\title{
Marden-Walker syndrome
}

INSERM

\section{Source}

INSERM. (1999). Orphanet: an online rare disease and orphan drug data base. MardenWalker syndrome. ORPHA:2461

Marden-Walker syndrome (MWS) is a malformation syndrome characterized by multiple joint contractures (arthrog ryposis), a mask-like face with blepharophimosis,

micrognathia, high-arched or cleft palate, low-set ears, decreased muscular bulk, kyphoscoliosis and arachnodactyly. 